\title{
Precise, three-dimensional seafloor geodetic deformation measurements using difference techniques
}

\author{
Peiliang $\mathrm{Xu}^{1}$, Masataka Ando ${ }^{2}$, and Keiichi Tadokoro ${ }^{2}$ \\ ${ }^{1}$ Disaster Prevention Research Institute, Kyoto University, Uji, Kyoto 611-0011, Japan \\ ${ }^{2}$ Research Center for Seismology and Volcanology, Graduate School of Science, Nagoya University, Nagoya 464-8502, Japan
}

(Received December 22, 2004; Revised June 8, 2005; Accepted June 30, 2005)

\begin{abstract}
Crustal deformation on land can now be measured and monitored routinely and precisely using space geodetic techniques. The same is not true of the seafloor, which covers about 70 percent of the earth surface, and is critical in terms of plate tectonics, submarine volcanism, and earthquake mechanisms of plate boundary types. We develop new data processing strategies for quantifying crustal deformation at the ocean floor: single- and double-difference methods. Theoretically, the single difference method can eliminate systematic errors of long period, while the double difference method is able to almost completely eliminate all depth-dependent and spatialdependent systematic errors. The simulations have shown that the transponders on the seafloor and thus the deformation of the seafloor can be determined with the accuracy of one centimeter in the single point positioning mode. Since almost all systematic errors (of temporal or spatial nature) have been removed by the double difference operator, the double difference method has been simulated to be capable of determining the threedimensional, relative position between two transponders on the seafloor even at the accuracy of sub-centimeters by employing and accumulating small changes in geometry over time. While the surveying strategy employed by the Scripps Institution of Oceanography (SIO) requires the ship maintain station, our technique requires the ship to move freely. The SIO approach requires a seafloor array of at least three transponders and that the relative positions of the transponders be pre-determined. Our approach directly positions a single transponder or relative positions of transponders, and thus measures deformation unambiguously.
\end{abstract}

Key words: Crustal deformation, seafloor geodesy, geodynamics.

\section{Introduction}

Geodetic deformation measurements have been important in computing crustal strains, inverting and understanding focal mechanisms of earthquakes, estimating plate motions, monitoring relative movement along faults and landslides, and detecting displacements due to volcanic magma flow (see, e.g. Frank, 1966; Ando, 1975; Prescott, 1981; Lambeck, 1988; Gordon and Stein, 1992). Crustal deformation can now be measured and monitored routinely and precisely using space geodetic techniques such as GPS and (In)SAR. This exciting scenario can only be seen on land unfortunately, since the L-band electromagnetic waves used by GPS and (In)SAR cannot penetrate sea water into seafloor. However, a great number of large earthquakes occur along the plate boundaries under the oceans, which cover about 70 percent of the whole surface of the Earth. Many great volcanoes also erupt under this unaccessible vast area of water. For instance, the three most important events, namely, the Tokai earthquakes, the Nankai earthquakes and the Tonankai earthquakes, which attract almost all the attention of seismologists in Japan and a vast of funding from the Japanese government, are all under water. Seafloor geodesy has been developed since the mid-

Copyright (c) The Society of Geomagnetism and Earth, Planetary and Space Sciences (SGEPSS); The Seismological Society of Japan; The Volcanological Society of Japan; The Geodetic Society of Japan; The Japanese Society for Planetary Sciences; TERRAPUB. 1980's thanks to advances in space geodesy and underwater acoustics, and is becoming an essential tool for understanding mechanisms of disastrous events under water, and hopefully, also in mitigating their effect on human being.

There are currently three major types of techniques for measuring deformation on the seafloor: (i) combination of kinematic GPS with underwater acoustics; (ii) seafloor tiltmeters; and (iii) seafloor seismometers. While the first two methods can detect deformation in long terms, the third method only records coseismic activity. In this paper, we will focus on the first type of techniques, since it can also be used as a seafloor tiltmeter if positioning is sufficiently precise and to detect deformation either due to plate motion, volcanoes and/or earthquakes. Another advantage of this type of techniques is that data are recorded on board of a ship and transponders on the seafloor respond only after being interrogated from the signal transmitted from the ship such that data is much easier to collect and the whole system can work longer. It cannot record coseismic deformation, however. For the other two types of techniques, the reader is referred to, e.g. Shimamura and Kanazawa (1988) and Anderson et al. (1997) for seafloor tiltmeters, and Webb (1998) and Webb, Deaton and Lemire (2001) for seafloor seismometers and their applications.

Seafloor geodetic positioning for seafloor crustal deformation measurement has been rapidly developed for almost two decades, thanks to great advancement in space geodesy 

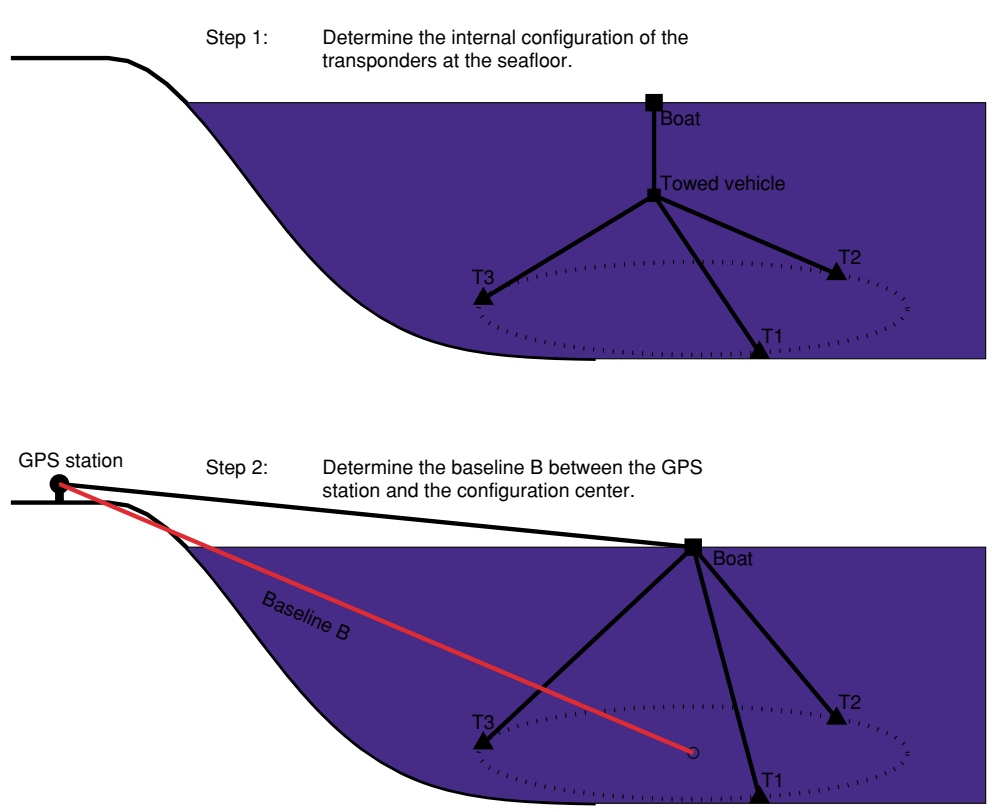

Fig. 1. Illustration of the SIO method. The upper plot has illustrated the determination of the internal configuration of the SIO method by towing a vehicle a few hundred meters above the seafloor, which consists of three transponders on the seafloor ideally distributed uniformly along a circle; the lower plot has shown the determination of the baseline marked in red by keeping the boat moving within a small area on the sea surface above the configuration center, and by using kinematic GPS and acoustic measurements.

and underwater acoustics (see e.g. Spiess, 1980, 1985a, b; Purcell et al., 1990; Fujimoto et al., 1995; Chadwell et al., 1998; Fujimoto et al., 1998; Spiess et al., 1998; Obana et al., 2000; Asada and Yabuki, 2001; Yamada et al., 2002; Chadwell, 2003). The technique consists of two basic components: (i) precise kinematic GPS; and (ii) precise underwater acoustics. Precise kinematic GPS serves three purposes in this seafloor geodetic positioning system: (i) to determine the position of the ship in real time; (ii) to determine the attitude of the ship for precise computation of the position of the transducer installed under the hull of the ship; and (iii) to connect the local seafloor geodetic network to the global reference frame. Underwater acoustics is fundamental in establishing a precise local seafloor geodetic network, however.

Seafloor geodetic positioning has been conducted in two different ways: (i) single point (transponder) positioning; and (ii) positioning the center of a configuration defined by three or four transponders. The starting observation equation for these two methods may be symbolically given in its simplest form as follows:

$$
\rho_{i j}=f\left(\mathbf{x}_{o_{i}}, \mathbf{x}_{j}\right)+\delta \rho_{d_{i j}}+\delta \rho_{v_{i j}}+\epsilon_{i j},
$$

where $\rho_{i j}$ is the ranging between the $i$ th transponder on the seafloor and the transducer under the ship, which can be calculated from the travel time and the sound velocity structure. $f()$ is a nonlinear functional of ray tracing, $\mathbf{x}_{o_{i}}$ is the unknown position of the $i$ th transponder. $\mathbf{x}_{j}$ is the position of the transducer, which can be directly calculated from kinematic GPS. $\delta \rho_{d_{i j}}$ is the systematic error due to the time delay in re-transmitting the received signal from the transponder back to the transducer, $\delta \rho_{v_{i j}}$ the systematic error due to the spatial and temporal variation in the sound velocity structure, in particular, in the area of upper 500 meters under the sea surface (Spiess, 1985a; Fujimoto et al., 1995; Chadwell et al, 1998; Lurton, 2002). This error varies from one observation to another, depending on both separation in time and space. $\epsilon_{i j}$ is the random ranging error. With modern transponders, $\delta \rho_{d_{i j}}$ would be at the level of millimeters and could be negligible, correspondingly, $\epsilon_{i j}$ is reported to be at the level of one centimeter (Chadwell et al., 1998). The most damaging factor in seafloor geodesy is due to $\delta \rho_{v_{i j}}$, which can reach some hundreds of ppm, or equivalently, the error of sound velocity in a few parts in $10^{4}$ (Spiess, 1985a).

In the case of single point positioning, Yamada et al. (2002) showed by simulations that the effect of $\delta \rho_{v_{i j}}$ on the position of a transponder can reach $18 \mathrm{~cm}$. In fact, this damaging effect was fully recognized in the very beginning of seafloor geodetic positioning. In order to eliminate the effect of this most important error source, the group of the Scripps Institution of Oceanography (SIO) has followed the proposal by Peter Bender, installed three or four transponders uniformly in a circle, kept the survey ship around the center of the configuration and determined the horizontal components of the position of the rigid polygon formed by transponders (see e.g. Spiess, 1985a; Chadwell et al., 1998; Spiess et al., 1998; Chadwell, 2003). We will refer to this positioning strategy as the SIO method in the rest of this paper. The SIO method is illustrated in Fig. 1 and consists of two basic steps: one to determine the internal configuration of the three (or four) transponders on the seafloor and the other to determine the baseline between the GPS station on land and the (non-physical) center of the polygon formed by the transponders. Due to the symmetry of the transponders and simultaneity of measurement of the ranges, $\delta \rho_{v_{i j}}$ are identical if the sound velocity is assumed to vary with depth only. As a result, it is clear mathematically that the effect of $\delta \rho_{v_{i j}}$ has cancelled out in the horizontal components of the central position. By collecting a large number of data 
and by assuming the relative positions of the transponders do not change in time, the repeatability of horizontal components was reported to be about $4 \mathrm{~cm}$ (Chadwell et al., 1998; Spiess et al., 1998) and more recently to be less than $10 \mathrm{~mm}$ (Gagnon et al., 2005). However, changes in the sound velocity directly affect the vertical component.

The major purpose of this paper is twofold: (i) to develop a new strategy to position a single transponder at the centimeter level of accuracy; and (ii) to investigate the feasibility to establish a seafloor geodetic network for precise seafloor deformation measurement. The paper is organized as follows. In Section 2, we propose a new single point positioning method by differencing the ranging measurements between two consecutive ship positions. A theoretical comparison with the SIO and other methods will be briefly given. In Section 3, we investigate the feasibility of establishing a seafloor geodetic network by combining the new differencing technique with more ranging and pressure data from a vehicle towed a few hundreds meters above the seafloor. In Sections 4 and 5, we will discuss the experiment design and carry out simulations to investigate the accuracy improvement of the new seafloor positioning method and the accuracy improvement of a seafloor geodetic network for precise seafloor deformation measurement.

\section{New Approach to Positioning a Single Transponder}

The most fundamental advantage of the SIO method over the current single point seafloor positioning is in that the SIO method is almost free of the effect of long and short period systematic error $\delta \rho_{v_{i j}}$ due to variations in sound velocities under the assumption that $\delta \rho_{v_{i j}}$ depends on water depth only. High precision in horizontal components is achieved by installing three or more transponders on the seafloor and by strictly controlling the survey vessel within a small area around the center of the circle defined by these three or four transponders. In this section we develop a technique to position a single transponder. This technique eliminates the long period systematic effect as does the SIO method. Short-period effects remain, but in a later section we show they are reduced by requiring the vessel to follow a symmetrical track around the transponder and then averaging these data or if the speed of the vessel is sufficiently fast such that the time between two measurements is much shorter than the period of these effects.

\subsection{Mathematical model}

We assume that the survey ship is at the positions $\mathbf{x}_{i}$ at time $t_{i}$ and $\mathbf{x}_{j}$ at time $t_{j} . \mathbf{x}_{i}$ and $\mathbf{x}_{j}$ are given, since they can be determined from kinematic GPS. We also assume that there is a single transponder on the seafloor, whose position $\mathbf{x}_{o}$ is to be determined from kinematic GPS and underwater acoustic ranging measurements $\rho_{s_{i} o}$ and $\rho_{s_{j} o}$. The surveying strategy is illustrated on the left part of Fig. 2. As in (1), we have two observation equations:

$$
\begin{gathered}
\rho_{s_{i} o}=f\left(\mathbf{x}_{o}, \mathbf{x}_{i}\right)+\delta \rho_{d_{s_{i} o}}+\delta \rho_{v_{s_{i} o}}+\epsilon_{i o}, \\
\rho_{s_{j} o}=f\left(\mathbf{x}_{o}, \mathbf{x}_{j}\right)+\delta \rho_{d_{s_{j} o}}+\delta \rho_{v_{s_{j} o}}+\epsilon_{j o},
\end{gathered}
$$

where $f\left(\mathbf{x}_{o}, \mathbf{x}_{i}\right)$ and $f\left(\mathbf{x}_{o}, \mathbf{x}_{j}\right)$ are the ranges computed from transponder and GPS positions, $\delta \rho_{d_{s_{i}}}$ and $\delta \rho_{d_{s_{j} o}}$ the systematic errors associated with the travel time, $\delta \rho_{v_{s_{i}} \text { ond }}$ and $\delta \rho_{v_{s_{j} o}}$ the systematic errors associated with the sound speed, $\epsilon_{i o}$ and $\epsilon_{j o}$ the combined random errors of the travel time and sound speed.

The linearized version of (2) is given as follows:

$$
\begin{gathered}
\rho_{s_{i} o}-f\left(\mathbf{x}_{o}^{0}, \mathbf{x}_{i}\right)=\mathbf{a}_{s_{i} o} d \mathbf{x}_{o}+\delta \rho_{d_{s_{i} o}}+\delta \rho_{v_{s_{i} o}} \\
+\epsilon_{i o}+\mathbf{b}_{s_{i} o} \epsilon_{x_{s i}}, \\
\rho_{s_{j} o}-f\left(\mathbf{x}_{o}^{0}, \mathbf{x}_{j}\right)= \\
\mathbf{a}_{s_{j} o} d \mathbf{x}_{o}+\delta \rho_{d_{s_{j} o}}+\delta \rho_{v_{s_{j} o}} \\
+\epsilon_{j o}+\mathbf{b}_{s_{j} o} \epsilon_{x_{s j}},
\end{gathered}
$$

where $\mathbf{x}_{o}^{0}$ is an approximate value of $\mathbf{x}_{o}, \mathbf{a}_{s_{i} o}$ and $\mathbf{a}_{s_{j} o}$ are the first partial derivatives of $f()$ with respect to $\mathbf{x}_{o}$ and computed with $\mathbf{x}_{o}^{0}, \mathbf{x}_{i}$ and $\mathbf{x}_{j}$, respectively. $d \mathbf{x}_{o}$ is the unknown coordinate correction vector to be estimated. $\mathbf{b}_{s_{i} o}$ and $\mathbf{b}_{s_{j} o}$ are the first partial derivatives with respect to $\mathbf{x}_{i}$ and $\mathbf{x}_{j}$, respectively. $\epsilon_{x_{s i}}$ and $\epsilon_{x_{s j}}$ are respectively the random errors of the survey vessel positions $\mathbf{x}_{i}$ and $\mathbf{x}_{j}$.

Applying the difference operator to two consecutive acoustic rangings by substracting ( $3 b)$ from (3a), we have:

$$
\Delta \rho_{i j}=\left(\mathbf{a}_{s_{i} o}-\mathbf{a}_{s_{j} o}\right) d \mathbf{x}_{o}+\Delta \rho_{d_{s_{i j} o}}+\Delta \rho_{v_{s_{i j} o}}+\Delta \epsilon_{i j o},
$$

where

$$
\begin{gathered}
\Delta \rho_{i j}=\rho_{s_{i} o}-f\left(\mathbf{x}_{o}^{0}, \mathbf{x}_{i}\right)-\rho_{s_{j} o}+f\left(\mathbf{x}_{o}^{0}, \mathbf{x}_{j}\right), \\
\Delta \rho_{d_{s_{i j} o}}=\delta \rho_{s_{s_{i} o}}-\delta \rho_{d_{s_{j} o}}, \\
\Delta \rho_{v_{s_{i j} o}}=\delta \rho_{v_{s_{i} o}}-\delta \rho_{v_{s_{j} o}}, \\
\Delta \epsilon_{i j o}=\epsilon_{i o}-\epsilon_{j o}+\mathbf{b}_{s_{i} o} \epsilon_{x_{s i}}-\mathbf{b}_{s_{j} o} \epsilon_{x_{s j}} .
\end{gathered}
$$

Now assume that the two vessel positions $\mathbf{x}_{i}$ and $\mathbf{x}_{j}$ are taken consecutively and are sufficiently close in space and time, then it is reasonable to believe that the two rays between the transducer and the transponder go through the same sound velocity structure. As a consequence, we should expect $\delta \rho_{v_{s_{i} o}} \approx \delta \rho_{v_{s_{j} o}}$. In other words, the third term $\Delta \rho_{v_{s_{i j}}}$ on the right hand side of (4a) should be equal or almost equal to zero. However, if the variation of sound velocity is of a short period, due to internal waves, for example, then the effect of $\Delta \rho_{v_{s_{i j}}}$ on the position of the transponder can still be significant. Therefore our observation equation retains an unknown bias term for each single difference observation. We will later show that the impact of this bias can be reduced by selecting a symmetrical ship track and averaging the data. On the other hand, since the ranging measurements $\rho_{s_{i} o}$ and $\rho_{s_{j} o}$ are taken on the same transponder, the time delays in re-transmitting the signals back to the transducer should remain unchanged as well. Thus the second term $\Delta \rho_{d_{s_{i j}}}$ on the right hand side of (4a) can be treated as zero.

Hence, given two consecutive and sufficiently close vessel positions $\mathbf{x}_{i}$ and $\mathbf{x}_{i+1}$, the observation equation (4a) can be simplified as follows:

$$
\Delta \rho_{i(i+1)}=\left(\mathbf{a}_{s_{i} o}-\mathbf{a}_{s_{i+1} o}\right) d \mathbf{x}_{o}+\Delta \epsilon_{i(i+1) o},
$$

if the variation of sound velocity in short periods can be neglected or if the speed of the ship is sufficiently fast such 


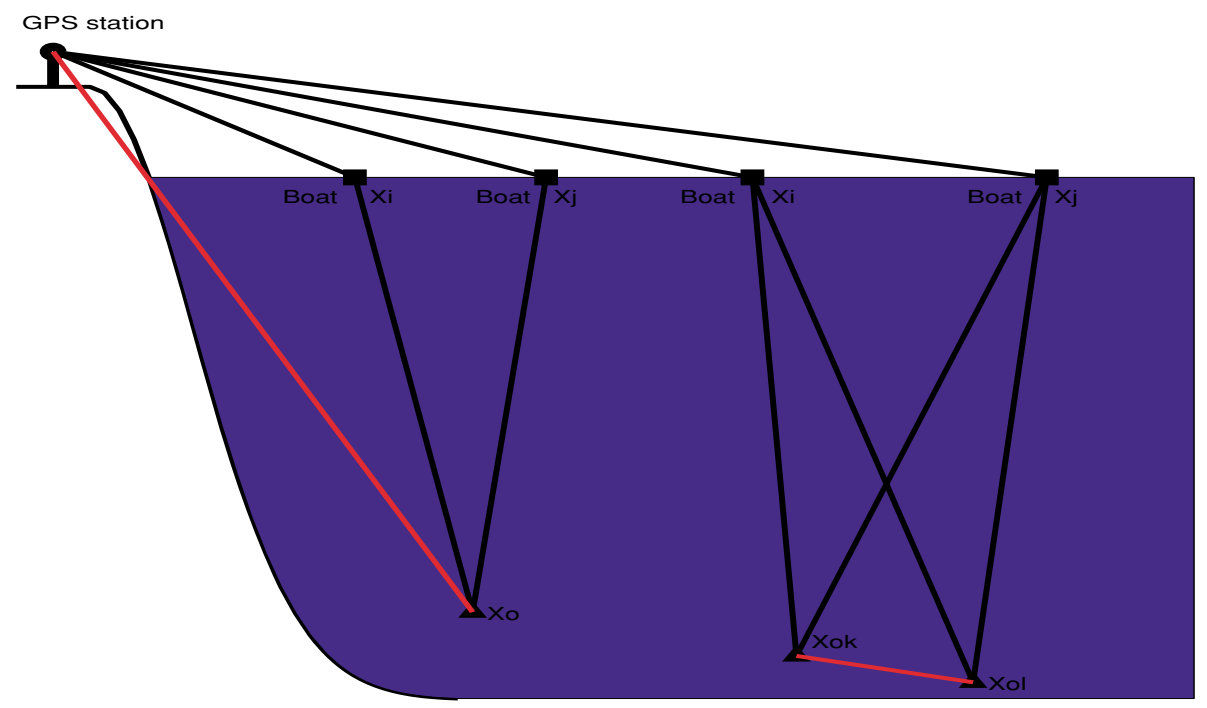

Fig. 2. Illustration of the new single- and double-difference methods. The left and right parts of the figure have illustrated the single- and double-difference processing strategies, respectively. In the single-difference mode, the major interest is in determining the baseline marked in red or simply the position $\mathbf{x}_{o}$ of the transponder on the seafloor; in the double-difference mode, the interest is in precisely determining the baseline between two transponders on the seafloor, which is also marked in red between $\mathbf{x}_{o_{k}}$ and $\mathbf{x}_{o_{l}}$.

that the time for the ship to move from one position to next is negligibly short compared with the short period effect. By collecting all the observation equations of this type together, we have the system of linear observation equations:

$$
\mathbf{y}=\mathbf{A} d \mathbf{x}_{o}+\epsilon_{y}
$$

It is obvious from (5) or (6) that the estimate of $d \mathbf{x}_{o}$ from the differenced ranging measurements is free of systematic errors of long wave nature. Although systematic errors of short wavelength may still retain due to the current practice of a slow ship speed, they will be negligible with advance of technology such that a ship can move sufficiently fast. As a result, we will not include the bias term in our equation.

\subsection{Stochastic model}

As in the single point positioning or the SIO method, the seafloor geodetic positioning system (6) is influenced by all the random error sources. Since (6) is based on differenced ranging measurements, the computed observations cannot be treated as independent. The variance-covariance matrices of the transducer positions at the times $t_{i}$ and $t_{j}$ are denoted by $\boldsymbol{\Sigma}_{s i}$ and $\boldsymbol{\Sigma}_{s j}$, respectively. Here the errors of the transducer position can be derived from those in kinematic GPS positioning, attitude determination and optical/laser surveys. The latter two techniques are needed to derive the position of transducer from the kinematic GPS position. Since optical/laser survey, for example, by using a total station, can be very precise at the millimeter level, its effect on the variance-covariance matrix could be negligible. According to Cohen (1996), the angular accuracy from attitude determination can be estimated by

$$
\sigma_{\theta}=\frac{0.5 \mathrm{~cm}}{L(\text { in cm })} .
$$

Given the antenna configuration of Chadwell (2003), the accuracies of roll and pitch would be about 1.17 minutes and 0.47 minutes, respectively. If one of the antennas is mounted within 10 meters from the transducer, then the correction error due to those of attitude determination will be a few millimeters, which is more than one magnitude smaller than the error of kinematic GPS positioning. Thus it is safe to simply replace the variance-covariance matrix of the transducer position with that from kinematic GPS positioning.

Further assume that the sound ranging measurements are stochastically independent and have an accuracy of $\sigma_{\rho}$. Then by applying the error propagation law to (4e), we can compute the variance of the differenced measurement:

$$
\sigma_{\Delta \rho_{i j}}^{2}=2 \sigma_{\rho}^{2}+\mathbf{b}_{s_{i} o} \boldsymbol{\Sigma}_{s i} \mathbf{b}_{s_{i} o}^{T}+\mathbf{b}_{s_{j} o} \boldsymbol{\Sigma}_{s j} \mathbf{b}_{s_{j} o}^{T}
$$

where the superscript $T$ stands for the transpose of a matrix or vector.

In the similar manner, for any two consecutive differenced ranging measurements $\Delta \rho_{i(i+1)}$ and $\Delta \rho_{(i+1)(i+2)}$, we can compute the covariance between them as follows:

$$
\begin{aligned}
& \operatorname{cov}\left(\Delta \rho_{i(i+1)}, \Delta \rho_{(i+1)(i+2)}\right) \\
& \quad=-\sigma_{\rho}^{2}-\mathbf{b}_{s_{(i+1)}} \boldsymbol{\Sigma}_{s(i+1)} \mathbf{b}_{s_{(i+1)}}^{T} .
\end{aligned}
$$

Because we have assumed that GPS positioning errors and sound speed errors are uncorrelated with time, $\Delta \rho_{i j}$ and $\Delta \rho_{k l}$ are uncorrelated if $k-j>1$. Under these assumptions the full variance-covariance matrix of the (differenced) observation vector $\mathbf{y}$ can be completely obtained from (8) and (9), which is denoted by $\boldsymbol{\Sigma}_{y}$ and is a positive definite matrix with a bandwidth of one only.

\subsection{Least squares estimate of $d \mathbf{x}_{o}$ and first compar- isons with other methods}

Applying the least squares method to (6), we can readily obtain the estimate of the position correction $d \mathbf{x}_{o}$ of the transponder on the seafloor as follows:

$$
\widehat{d \mathbf{x}}_{o}=\left(\mathbf{A}^{T} \boldsymbol{\Sigma}_{y}^{-1} \mathbf{A}\right)^{-1} \mathbf{A}^{T} \boldsymbol{\Sigma}_{y}^{-1} \mathbf{y}
$$


from which we can compute the final transponder position

$$
\hat{\mathbf{x}}_{o}=\mathbf{x}_{o}^{0}+\widehat{d \mathbf{x}}_{o}
$$

and its variance-covariance matrix $\boldsymbol{\Sigma}_{\hat{x}_{o}}$

$$
\boldsymbol{\Sigma}_{\hat{x}_{o}}=\left(\mathbf{A}^{T} \boldsymbol{\Sigma}_{y}^{-1} \mathbf{A}\right)^{-1} \text {. }
$$

Comparing (10) with the single transponder positioning in the literature (see e.g. Yamada et al., 2002), we see that the new approach (10) is free of long term systematic effects. Short term biases may retain, unless the ship moves sufficiently fast. If the number of differenced measurements has been accumulated such that sufficient strength of geometry $\mathbf{A}$ is obtained and that short term effects can be averaged to zero, then one centimeter accuracy of position can be obtained for the transponder on the seafloor, which will be confirmed in Section 5 by simulations.

Comparing (10) with the SIO method, we see that both methods are not affected in the horizontal components of the transponder position by systematic errors due to heterogeneity in the sound velocity structure. Unlike the SIO method, the new positioning method can also produce the precise vertical component. Neither approach is biased by time delay in the re-transmission of signal from the transponder. The new approach differences this away by assuming that the magnitude of the delay does not change appreciably as the aspect and azimuth angle at the transducer changes from the first to the second range used to form the difference. The SIO approach keeps the same aspect and azimuth angle between the shipboard and seafloor transponders so this bias is constant and is cancelled out in determining the polygon center of the array. The new method positions a single transponder by developing a new data processing strategy and by requiring the survey vessel to either move freely if the speed of the vessel moves sufficiently fast or to maneuver along a symmetrical track around the transponder in the current situation of slow ship speed. The SIO method uses 3 or 4 transponders and requires the ship hold station at the center of the transponder array. The new method measures the displacement of a single transponder while the SIO method measures the displacement of the patch of seafloor defined by the polygon formed by the transponders. Thus the new method can provide a finer spatial resolution of the deformation field. This can be of particular importance in regions with significant local strain, where the SIO method must collect data to re-determine the internal configuration of the transponders. The SIO method is free of long and short period errors in sound speed, while the new single difference method requires a to-be-determined track to be driven to accumulate enough changes in geometry such that the average effect of biases is near zero, unless the ship moves sufficiently fast such that the time between two ship positions is sufficiently short to neglect the short period effect.

\section{Seafloor Geodetic Network}

Given a seafloor geodetic network defined by a number of transponders, we could use the single positioning method described in the previous section to precisely determine the positions of all the transponders. With the rapid advancement of technology, precise measurements other than arrival times between the transducer on the survey vessel and the transponders on the seafloor are expected. For instance, in the case of the SIO method, a lot more of ranging measurements have been collected by towing a measuring unit at about a few hundreds meters above the seafloor. In addition, height differences between the hydrophone mounted on the towed unit and the transponders can also be computed (see, e.g., Spiess, 1985b; Chadwell et al., 1998; Spiess et al., 1998; Sweeney et al., 2005). Fujimoto et al. $(1995,1998)$ also did a lot of experiments on measuring the distance between two transponders on the seafloor and reported that if poor data are removed, the ranging error would reach one or two centimeters.

In this section, we will combine these measurements with usual ranging measurements between the survey vessel and transponders on the seafloor described and used in the previous section into a seafloor geodetic network, and investigate how these extra measurements will improve the accuracy of seafloor geodetic positioning. Given a seafloor geodetic network with $m$ transponders properly distributed on the seafloor, assuming that in addition to the ranging measurements between the transducer mounted on the survey vessel and the transponders on the seafloor, we also collect the height differences and ranging measurements through the towed hydrophone at about a few hundreds meters above the seafloor.

\subsection{Measurements collected by towed hydrophones}

Since the observation equations for the measurements between the vessel and the transponders have been given in the previous section, we will focus on the height differences and ranging measurements collected by the towed hydrophone here. Assume that the towed hydrophone at the time $t_{i}$ collect data from a subset of $m_{i}$ transponders on the seafloor, which is denoted by $\left(1,2, \ldots, m_{i}\right)$. Then for any height difference $h_{i j}$ between the towed hydrophone and the $j$ th transponder, we have the observation equation:

$$
h_{i o_{j}}=H\left(\mathbf{x}_{i}, \mathbf{x}_{o_{j}}\right)+\epsilon_{h_{i j}}, \quad j \in\left(1,2, \ldots, m_{i}\right)
$$

where $H(\cdot)$ is the functional defined by ray tracing, $\mathbf{x}_{i}$ is the position of the towed hydrophone at the time $t_{i}$, which is unknown and to be determined. $\mathbf{x}_{o_{j}}$ is the unknown position of the $j$ th transponder or station of the seafloor geodetic network. $\epsilon_{h_{i j}}$ is the observation error of $h_{i j}$. According to Spiess (1985b) and Spiess et al. (1998), the accuracy of $h_{i o_{j}}$ would be about 5 centimeters.

Linearizing (11) results in the equations:

$$
\begin{array}{r}
h_{i o_{j}}-H\left(\mathbf{x}_{i}^{0}, \mathbf{x}_{o_{j}}^{0}\right)=\mathbf{c}_{i o_{j}} d \mathbf{x}_{o_{j}}+\mathbf{g}_{i o_{j}} d \mathbf{x}_{i}+\epsilon_{h_{i j}}, \\
j \in\left(1,2, \ldots, m_{i}\right)
\end{array}
$$

where $\mathbf{x}_{i}^{0}$ and $\mathbf{x}_{o_{j}}^{0}$ are approximate values of $\mathbf{x}_{i}$ and $\mathbf{x}_{o_{j}}$, respectively. $d \mathbf{x}_{o_{j}}$ is the the correction of $\mathbf{x}_{o_{j}}$ and $d \mathbf{x}_{i}$ that of $\mathbf{x}_{i} \cdot \mathbf{c}_{i o_{j}}$ and $\mathbf{g}_{i o_{j}}$ are the partial derivatives of $H(\cdot)$ with respect to $\mathbf{x}_{o_{j}}$ and $\mathbf{x}_{i}$, respectively.

Similarly to (3a), and bearing in mind that unlike $\mathbf{x}_{i}$ of (3a), $\mathbf{x}_{i}^{0}$ is not random but unknown to be determined, the ranging measurement between the towed hydrophone and 
the $j$ th transponder can be linearized as follows:

$$
\begin{aligned}
\rho_{i o_{j}}-f\left(\mathbf{x}_{o_{j}}^{0}, \mathbf{x}_{i}^{0}\right)= & \mathbf{a}_{i o_{j}} d \mathbf{x}_{o_{j}}+\mathbf{b}_{i o_{j}} d \mathbf{x}_{i}+\delta \rho_{d_{i o_{j}}}+\delta \rho_{v_{i o_{j}}} \\
& +\epsilon_{i o_{j}}, \quad j \in\left(1,2, \ldots, m_{i}\right)
\end{aligned}
$$

Here $\mathbf{a}_{i o_{j}}$ and $\mathbf{b}_{i o_{j}}$ are the partial derivatives of $f(\cdot)$ with respect to $\mathbf{x}_{o_{j}}$ and $\mathbf{x}_{i}$, respectively. Since the systematic errors due to the heterogeneity of sound velocity structure at deep sea and the time delay are rather small, if they are neglected from (13), then we have

$$
\begin{array}{r}
\rho_{i o_{j}}-f\left(\mathbf{x}_{o_{j}}^{0}, \mathbf{x}_{i}^{0}\right)=\mathbf{a}_{i o_{j}} d \mathbf{x}_{o_{j}}+\mathbf{b}_{i o_{j}} d \mathbf{x}_{i}+\epsilon_{i o_{j}}, \\
j \in\left(1,2, \ldots, m_{i}\right)
\end{array}
$$

However, as a remedy, although the noise of ranging measurements could reach one $\mathrm{cm}$ or be better, the accuracy of ranging measurements and height differences has been set to 10 and $5 \mathrm{~cm}$, respectively (see, e.g. Spiess, 1985b; Spiess et al., 1998).

Collecting all the linearized observation equations (12) and (14) through the whole seafloor geodetic network together, we have the final system of linearized observation equations:

$$
\mathbf{y}_{t}=\mathbf{A}_{t} d \mathbf{X}+\boldsymbol{\epsilon}_{t},
$$

where $\mathbf{y}_{t}$ stands for all the values on the left hand sides of (12) and (14) taken from the towed unit, $\mathbf{A}_{t}$ is the coefficient matrix, $\boldsymbol{\epsilon}_{t}$ is the error vector of $\mathbf{y}_{t}$, and

$$
d \mathbf{X}=\left\{d \mathbf{x}_{o_{1}}^{T}, d \mathbf{x}_{o_{2}}^{T}, \ldots d \mathbf{x}_{o_{m}}^{T}, d \mathbf{x}_{1}^{T}, d \mathbf{x}_{2}^{T}, \ldots, d \mathbf{x}_{q}^{T}\right\}^{T} .
$$

Here $q$ is the total number of time epoches of data taken by the towed unit.

In the single point positioning mode, all the positions of transponders can be independently determined, for which we only need to solve a $(3 \times 3)$ normal matrix. This is not true any more in the case of (15). Putting all the observation equations (6) and (15) together, we are ready to solve the seafloor geodetic network. In the network mode, we have to solve a $\{3(m+q) \times 3(m+q)\}$ normal matrix, since all the unknowns $d \mathbf{x}_{o_{i}}\{i \in(1,2, \ldots, m)\}$ and $d \mathbf{x}_{j}\{j \in$ $(1,2, \ldots, q)\}$ are intermingled together through (12) and (14).

\subsection{More difference operators for use in the network mode}

Alternatively, instead of using the difference (4a) between two ranging measurements taken at different time epoches, in the network mode, we can also difference two ranging measurements between different transponders taken at the same time epoch. Assume that the vesselmounted hydrophone at the time $t_{i}$ collect data from a subset of $m_{i}$ transponders on the seafloor, which is denoted by $\left(1,2, \ldots, m_{s i}\right)$. In a similar manner to (3a), we have

$$
\begin{aligned}
\rho_{i o_{k}}-f\left(\mathbf{x}_{o_{k}}^{0}, \mathbf{x}_{i}\right)= & \mathbf{a}_{i o_{k}} d \mathbf{x}_{o_{k}}+\delta \rho_{d_{i o_{k}}}+\delta \rho_{v_{i o_{k}}} \\
& +\epsilon_{i o_{k}}+\mathbf{b}_{i o_{k}} \epsilon_{x_{i}}, \\
\rho_{i o_{l}}-f\left(\mathbf{x}_{o_{l}}^{0}, \mathbf{x}_{i}\right)= & \mathbf{a}_{i o_{l}} d \mathbf{x}_{o_{l}}+\delta \rho_{d_{i o_{l}}}+\delta \rho_{v_{i o_{l}}} \\
& +\epsilon_{i o_{l}}+\mathbf{b}_{i o_{l}} \epsilon_{x_{i}},
\end{aligned}
$$

for any two transponders $k, l \in\left(1,2, \ldots, m_{s i}\right)$ (compare the right part of Fig. 2).
Applying the difference operator to (16) by taking (16b) from (16a), we have the new difference ranging measurement

$$
\nabla \rho_{k l}^{i}=\mathbf{a}_{i o_{k}} d \mathbf{x}_{o_{k}}-\mathbf{a}_{i o_{l}} d \mathbf{x}_{o_{l}}+\nabla \rho_{d_{k l}}^{i}+\nabla \rho_{v_{k l}}^{i}+\nabla \epsilon_{k l}^{i},
$$

where

$$
\begin{gathered}
\nabla \rho_{k l}^{i}=\rho_{i o_{k}}-\rho_{i o_{l}}-f\left(\mathbf{x}_{o_{k}}^{0}, \mathbf{x}_{i}\right)+f\left(\mathbf{x}_{o_{l}}^{0}, \mathbf{x}_{i}\right), \\
\nabla \rho_{d_{k l}}^{i}=\delta \rho_{d_{i o_{k}}}-\delta \rho_{d_{i o_{l}}}, \\
\nabla \rho_{v_{k l}}^{i}=\delta \rho_{v_{i o_{k}}}-\delta \rho_{v_{i o_{l}}}, \\
\nabla \rho_{d_{k l}}^{i}=\epsilon_{i o_{k}}-\epsilon_{i o_{l}}+\left(\mathbf{b}_{i o_{k}}-\mathbf{b}_{i o_{l}}\right) \epsilon_{x_{i}} .
\end{gathered}
$$

As in the case of single point positioning, (17d) would be equal to zero, if the variation in sound velocity is assumed to be depth-dependent within the circle with radius of a few kilometers. Since the difference is taken at the same time epoch, variations with a short period of few minutes to hours such as effect of potential internal waves will also be eliminated by the difference operator (17d). Nevertheless, if the sound speed structure is horizontally stratified, the spatial effect will retain and (17d) will reflect this effect. However, since $\nabla \rho_{d_{k l}}^{i}$ is involved with two transponders, the effect of time delays cannot be eliminated, unless they are negligible.

In a similar manner to (17a), we can write another difference measurement for the time epoch $t_{j}$ as follows:

$$
\nabla \rho_{k l}^{j}=\mathbf{a}_{j o_{k}} d \mathbf{x}_{o_{k}}-\mathbf{a}_{j o_{l}} d \mathbf{x}_{o_{l}}+\nabla \rho_{d_{k l}}^{j}+\nabla \rho_{v_{k l}}^{j}+\nabla \epsilon_{k l}^{j} .
$$

As in (17a), $\nabla \rho_{v_{k l}}^{j}$ (if not zero) reflects the effect of a horizontally stratified structure of sound speed. Now applying the difference operator further to (17a) and (18), we have the double-difference measurement

$$
\begin{aligned}
\nabla \Delta \rho_{k l}^{i j}= & \left(\mathbf{a}_{i o_{k}}-\mathbf{a}_{j o_{k}}\right) d \mathbf{x}_{o_{k}}-\left(\mathbf{a}_{j o_{k}}-\mathbf{a}_{j o_{l}}\right) d \mathbf{x}_{o_{l}} \\
& +\nabla \Delta \rho_{v_{k l}}^{i j}+\nabla \Delta \epsilon_{k l}^{i j},
\end{aligned}
$$

where

$$
\begin{gathered}
\nabla \Delta \rho_{k l}^{i j}=\nabla \rho_{k l}^{i}-\nabla \rho_{k l}^{j}, \\
\nabla \Delta \rho_{v_{k l}^{i j}}^{i j}=\nabla \rho_{v_{k l}}^{i}-\nabla \rho_{v_{k l}}^{j}, \\
\nabla \Delta \epsilon_{k l}^{i j}=\nabla \epsilon_{k l}^{i}-\nabla \epsilon_{k l}^{j} .
\end{gathered}
$$

The time delays has been completely eliminated in the double-difference measurement and thus have no effect on positioning.

If the two consecutive positions of the ship are sufficiently close, $\nabla \rho_{v_{k l}}^{i}$ and $\nabla \rho_{v_{k l}}^{j}$ cancel out each other, and $\nabla \Delta \rho_{v_{k l}}^{i j}$ is equal to zero. Thus (19) can be further simplified as follows:

$$
\nabla \Delta \rho_{k l}^{i j}=\left(\mathbf{a}_{i o_{k}}-\mathbf{a}_{j o_{k}}\right) d \mathbf{x}_{o_{k}}-\left(\mathbf{a}_{j o_{k}}-\mathbf{a}_{j o_{l}}\right) d \mathbf{x}_{o_{l}}+\nabla \Delta \epsilon_{k l}^{i j} .
$$

The double difference operator will result in the decrease of number of observations for the determination of the positions of seafloor transponders, however. Thus if transponders are sufficiently precise with negligible time delay and if systematic errors are of long wave nature, then single difference is preferred to double difference. Otherwise, the 
advantage of applying double difference should be obvious. Similarly, one can also apply these difference operators to the measurements collected from the towed hydrophone(s), which is omitted here.

By combining (20) with the equations of type (5) and those from the towed hydrophone(s), and by using the method of previous section to derive the a priori variancecovariance matrix, we can apply the least squares method to solve for all the positions of the transponders on the seafloor in the network mode.

\section{Experiment Design}

We will design a number of experiments in this section to demonstrate the attainable accuracy by the new difference methods for a single point seafloor positioning and network mode, and potential accuracy improvement by including more data in the network mode. Since geometric topologies of ray paths in the oceanic environment are similar or only slightly different between the depth-dependent and constant speed structures (Lurton, 2002), we will simply use the latter for establishing observation equations in our simulations for simplicity and focus on the effect of systematic and random errors on seafloor geodetic positioning. This simplification may be sufficient for our accuracy simulation, since the sound velocity variability due to internal waves has negligible effect on the take-off angle (Flatté and Vera, 2002), and as a result, also negligible effect on the coefficients of the observation equations in the previous sections. In practical applications, we should write observation equations based on Snell's law of refraction and compute the position of the transponder on the seafloor or adjust the seafloor geodetic network by using a modelled or measured sound velocity structure, however.

The experiment design for seafloor geodetic positioning consists mainly of four parts: (i) design of positioning modes, namely, single point positioning or network; (ii) selection of difference operators; (iii) models for systematic errors in ranging measurements; and (iv) models for random errors of GPS/acoustic measurements. We design two single point positioning experiments with different sample spacings along the trajectory of the survey vessel, respectively. We also design two relative seafloor positioning experiments- the simplest geodetic networks on the seafloor, whose stations (transponders) are separated by $1 \mathrm{~km}$, respectively. Since measurements in network mode are collected from, at least, a few hundreds meters above the seafloor, the shape of network triangles can be best chosen practically without loss of generality. Keeping in mind possible direct applications of the simulation results in this paper to monitoring the source area of the Nankai earthquakes, we decide to choose the water depth of $3000 \mathrm{~m}$. In order to avoid or minimize the effect of multipath, the vessel is allowed to move within a square area with length roughly equal to the depth of water.

In order to simulate systematic errors $\delta \rho_{v}$, we consider in Eq. (21) the four types of effects: (i) a constant term; (ii) internal wave with a short period; (iii) diurnal and/or (semi-)diurnal tides; and (iv) factors of regional effect by using a Gaussian correlation function. More specifically, we simulate the systematic errors in centimeters as follows:

$$
\begin{aligned}
\delta \rho_{v}= & c_{1}+c_{2} \sin \left(\frac{2\left(t-t_{0}\right)_{m}}{T_{w}} \pi\right)+c_{3} \sin \left(\frac{\left(t-t_{0}\right)_{h}}{12} \pi\right) \\
& +c_{4}\left[1-\exp \left\{-\frac{1}{2}\left\|\mathbf{x}-\mathbf{x}^{\prime}\right\|^{2} /(2 \mathrm{~km})^{2}\right\}\right] .
\end{aligned}
$$

Since the effect at the mesoscale of hundreds and/or thousands $\mathrm{km}$ should produce the same effect over a small area, we may attribute such an effect to the first constant $c_{1}$. According to Flatté et al. (1979) and Colosi et al. (1994), the effect of internal wave on sound velocity could be five parts in $10^{4}$ at the surface and one part in $10^{4}$ at the depth of 1 $\mathrm{km}$. Although internal wave has been always treated as a random phenomenon in ocean acoustics (see e.g. Flatté et al., 1979; Colosi et al., 1994; Desaubies, 1990; Flatté and Vera, 2002), we have to treat it as a source of systematic errors and have to remove or eliminate its effect on seafloor positioning for precise seafloor deformation measurement. A diurnal error with an amplitude of about $20 \sim 30 \mathrm{~cm}$ can also be seen clearly in Spiess et al. (1998). Taking all these into account, we have chosen the four constants $c_{1}=10, c_{2}=12$ as in Yamada et al. (2002), $c_{3}=30$ and $c_{4}=2$. The time units in the second and third terms on the right hand side are in minutes and hours, respectively, with $T_{w}=20$. $\left\|\mathbf{x}-\mathbf{x}^{\prime}\right\|$ is the length between the points $\mathbf{x}$ and $\mathbf{x}^{\prime}$. Three ranging errors of 1,5 and $7 \mathrm{~cm}$ will be used in our simulations, and that of height differences is assumed to be $5 \mathrm{~cm}$. The accuracy of GPS kinematic positioning is assumed to be $10 \mathrm{~cm}$. We should note that if the ship is far away from land such that GPS integer ambiguity unknowns cannot be correctly fixed, then kinematic GPS will not be able to provide the position accuracy of $10 \mathrm{~cm}$. In this case, one may have to either consider using some intermediate means (one more ship, for example) to bridge land GPS stations and the surveying vessel or simply treat the seafloor geodetic network as a free network. Since we do not have the a priori information on the correlations among the three components, we simply assume $\boldsymbol{\epsilon}_{x}$ is normally distributed with mean zero and variance-covariance matrix $\sigma_{x}^{2} \mathbf{I}$, where $\mathbf{I}$ is an identity matrix and $\sigma_{x}=10 / \sqrt{3} \mathrm{~cm}$.

\section{Simulation Results}

\subsection{Single point positioning}

5.1.1 Positioning by using the SIO method As the first simulation, we will investigate the effect of random and systematic errors on the SIO method. Following Spiess (1985a, b) and Spiess et al. (1998), we assume that three transponders are uniformly installed on the seafloor along the circle with a diameter equal to the depth of the water. The survey vessel is allowed to freely move horizontally within the circular area with a radius of 200 meters from the center of the transponder configuration. The free movement is simulated by using a process of random walk with a step of 20 meters. In total, we have simulated about 5800 samples. The effect of systematic errors (21) on the SIO method has been shown in Fig. 3. It is very clear from the red and black lines that the SIO method is indeed able to accurately determine the horizontal components of the center of the transponder configuration, since the effect of 


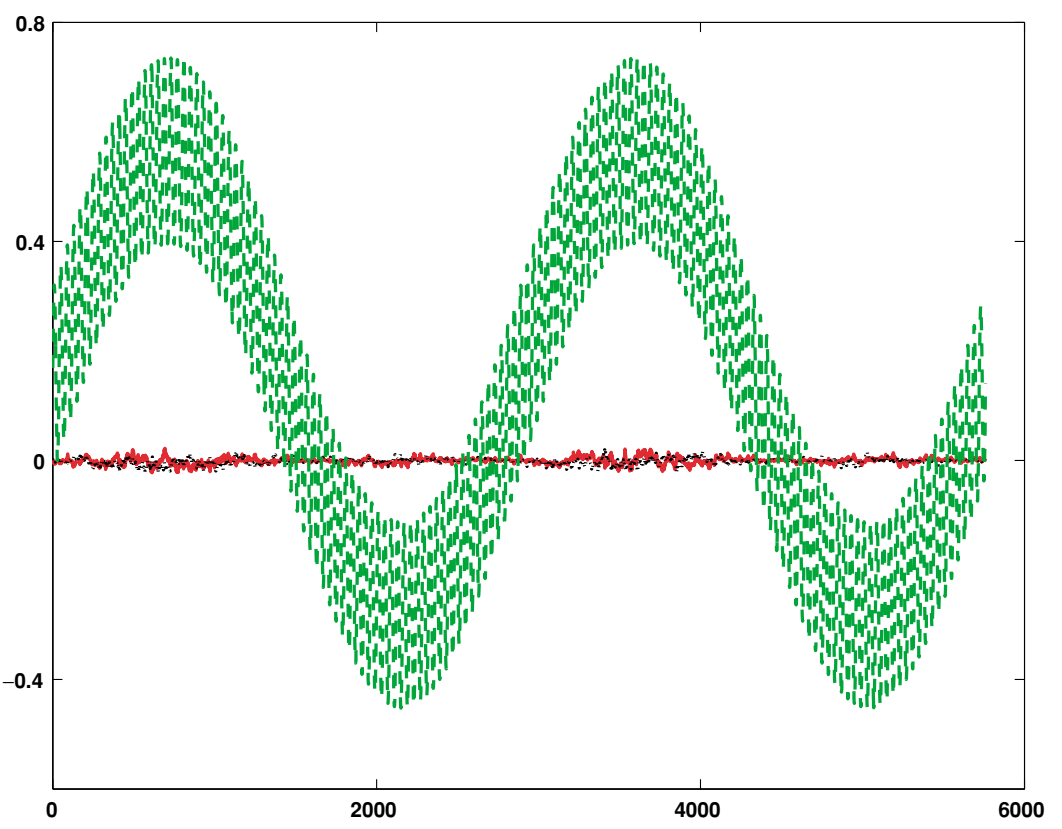

Fig. 3. The effects of systematic errors on the SIO method (unit: meters). The horizontal axis marks the sampling epochs. The effects of systematic errors on the SIO method are shown in the red solid and black dotted lines (the horizontal components) and the green dashed line (the vertical component).

the simulated systematic errors on these two components is close to zero. However, the effect of the systematic errors on the vertical component (the green line in Fig. 3) remains uncancelled and is significantly large, varying from -0.452 to $0.735 \mathrm{~m}$ with an average effect of $0.141 \mathrm{~m}$.

We have to note that almost zero effect of systematic errors on the horizontal components reported above should not be confused with or interpreted as the final possible (extremely high) accuracy in the horizontal components by the SIO method, for two reasons: (i) random measurement errors and the effect of spatial variations in sound speed are not included in the above confirmation of the ability of the SIO method to remove the systematic effect of depthdependent effect; and (ii) in order to derive the final positioning accuracy, we have to consider the effect of the uncertainty in the internal configuration of the three transponders uniformly distributed along a circle on the seafloor. In fact, the errors of the configuration center can be mathematically written as follows:

$$
\boldsymbol{\epsilon}_{c}^{i}=\boldsymbol{\epsilon}_{g p s}^{i}-\boldsymbol{\epsilon}_{\rho}^{i}-\mathbf{b}_{\rho}^{i}+\boldsymbol{\epsilon}_{x_{t}},
$$

for each epoch of measurement, where the superscript stands for the $i$ th epoch, $\boldsymbol{\epsilon}_{c}^{i}$ is the error vector of the derived configuration center, $\epsilon_{g p s}^{i}$ is due to the errors of kinematic GPS, $\epsilon_{\rho}^{i}$ is due to the random errors of ranges between the transponders and the transducer, $\mathbf{b}_{\rho}^{i}$ is the systematic errors of the derived configuration center due to (21), and $\boldsymbol{\epsilon}_{x_{t}}$ is due to the errors of the internal configuration of the transponders. What we have shown in Fig. 3 are actually the bias term of $\mathbf{b}_{\rho}^{i}$ with the changes of epochs. Almost zero average effect of the systematic errors on the horizontal components can be obviously observed. Since the first two terms on the right hand side of (22) are random and are dependent on the time epochs, their contribution to the errors of the final averaged configuration center could be negligible if the number of epochs is sufficiently large. The effect of the errors in the internal configuration certainly does not change with epochs. If the relative positions of the transponders do not change throughout the experiment, then the biases cancel out. Using real data, Gagnon et al. (2005) have shown that the repeatability of the SIO method can be less than $10 \mathrm{~mm}$. If the relative positions of the transponders are likely to change, then they can be re-measured with a resolution of $2 \mathrm{~cm}$ using a deeply towed vehicle (Sweeney et al., 2005). However, if there exist spatial variations in the sound velocity structure, the (horizontal) accuracy of the SIO method would deteriorate, the extent of which depends on the relative magnitude of the spatial variations.

5.1.2 Positioning by the difference method We will now design a number of experiments to study the effectiveness of the difference method for precise seafloor positioning. More specifically, we will investigate the effects of different factors on the single seafloor positioning by the difference method. These factors include: (i) differences in the accuracy of observations; (ii) short period of internal waves; (iii) sampling spacings; and (iv) different surveying strategies, which are shown in Fig. 4.

Given the vessel speed of $1 \mathrm{~m} / \mathrm{s}$ and the sampling period of about 1.667 minutes for the circular surveying trajectory, we plotted in Fig. 5 the corresponding original systematic errors in $\rho_{s_{i} o}$ and those remaining in the differenced observations $\Delta \rho_{i j}$, with and without the effect of internal waves of short period. In the case of no internal waves, namely, $c_{2}$ in (21) is equal to zero, then the remaining systematic errors have been almost completely cancelled out (compare the black dash-dotted line in Fig. 5), changing from -2.2 to $2.2 \mathrm{~mm}$ with an average of $0.1 \mathrm{~mm}$. If the effect of internal waves is included, the original systematic errors are between -31.99 and $51.99 \mathrm{~cm}$. However, it is clear from the 

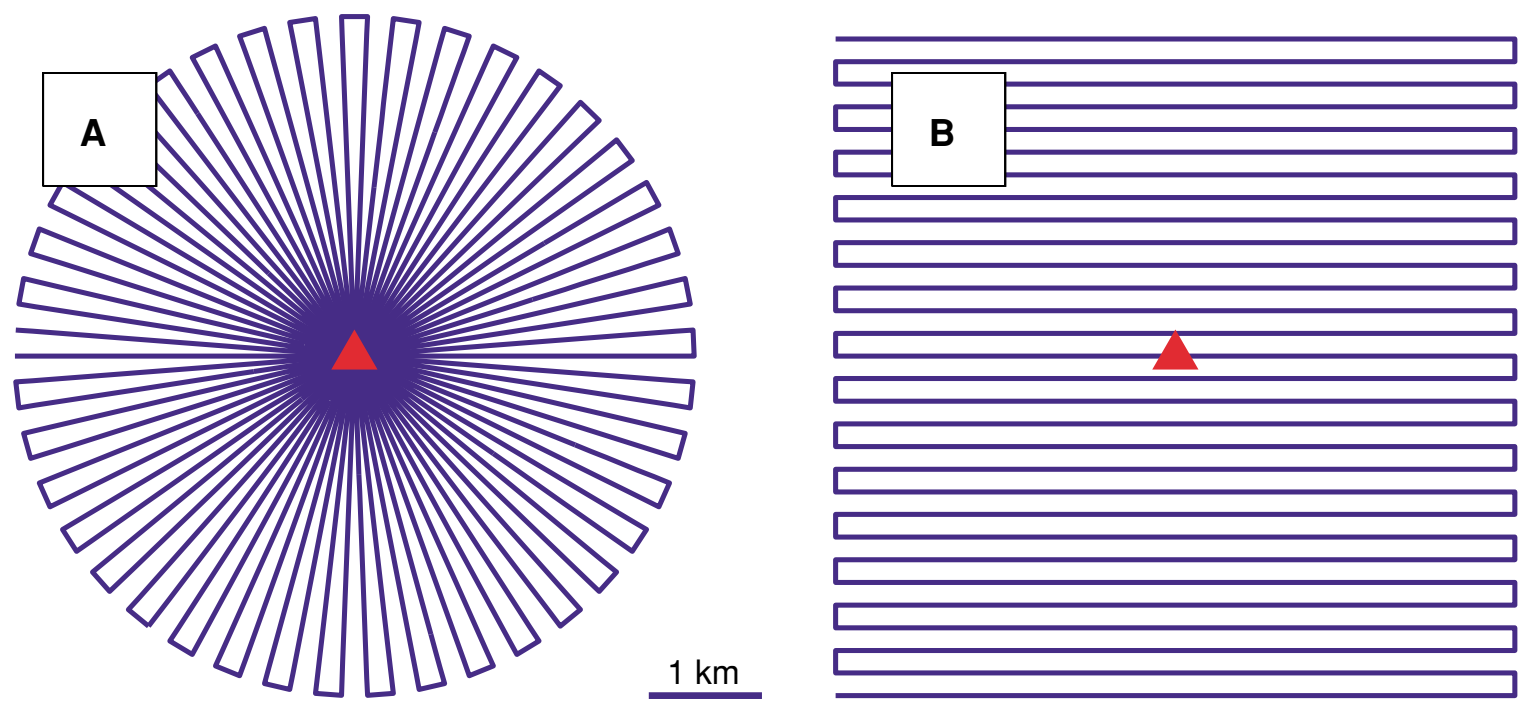

Fig. 4. The two surveying strategies (trajectories) for testing the performance of the difference method: the solid lines—-the surveying trajectories; the red triangles - the transponders on the seafloor.

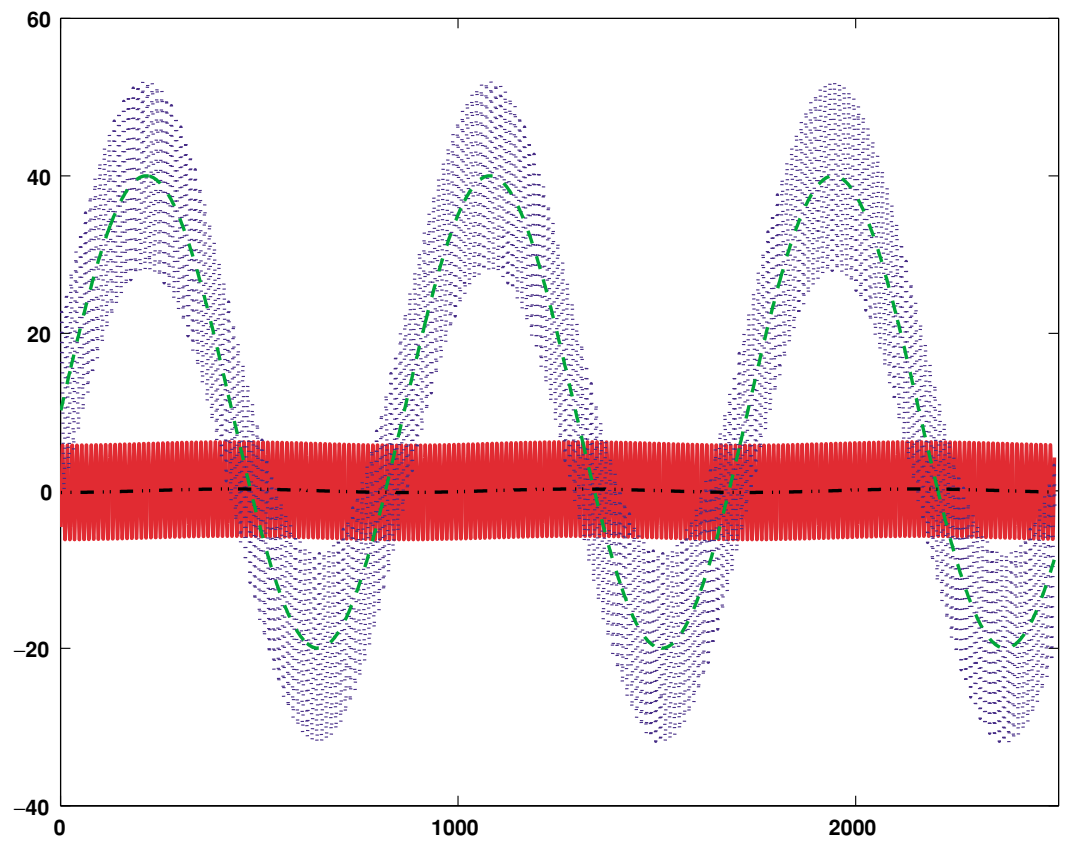

Fig. 5. The systematic errors of the original and differenced acoustic ranging measurements, with and without the effects of internal waves of short period. The horizontal axis marks the sampling epochs. The vertical axis is marked in centimeters. The original systematic errors are shown in the blue dotted and green dashed lines, with and without the effect of internal waves, respectively. The remaining systematic errors in the differenced acoustic ranging measurements are in the red solid and black dash-dotted lines with and without the effect of internal waves, respectively.

red line in Fig. 5 that a significant part of systematic errors in the original acoustic ranging measurements still remains in the differenced measurements, varying from -6.22 to $6.22 \mathrm{~cm}$ with an average of $0.1 \mathrm{~mm}$, basically due to the effect of internal waves of short period. Listed in Table 1 are the accuracy results of positioning from the circular (A) trajectory (compare Fig. 4) by using the difference method, with different accuracy of 1,5 and $7 \mathrm{~cm}$ for acoustic ranging measurements $\rho_{s_{i} o}$, and with and without the effect of internal waves of short period taken into account.

We can see from Table 1 that the difference method can result in subcentimeter level of accuracy in the horizontal components and centimeter level of accuracy in the vertical component, no matter whether there is the effect of internal waves of short period, even though the systematic errors in the original acoustic ranging measurements are very large. If no internal waves exist or if systematic errors are not of short period, we have seen from the black dash-dotted line of Fig. 5 that the difference method has eliminated almost completely the systematic errors of the raw measurements, and as a result, should not be affected by the systematic errors in this case. Although a significant part of systematic errors remains in the differenced measurements, the experiments have shown that a well-designed (symmetrical) sur- 
Table 1. The accuracy of the seafloor positioning from the circular surveying trajectory by using the difference method, with different acoustic ranging accuracy of 1,5 and $7 \mathrm{~cm}$, and with and without the effect of internal waves of short period. Horizontal and Vertical in the table stand for the horizontal and vertical components of the seafloor transponder position, respectively.

\begin{tabular}{|c|c|c|c|c|c|}
\hline \multirow{2}{*}{\multicolumn{2}{|c|}{$\begin{array}{l}\text { Position Accuracy } \\
\text { Ranging Accuracy }\end{array}$}} & \multicolumn{3}{|c|}{ Total MSE $(\mathrm{cm})$} & \multirow{2}{*}{$\begin{array}{l}\text { Systematic } \\
\text { Effect }(\mathrm{cm})\end{array}$} \\
\hline & & $7 \mathrm{~cm}$ & $5 \mathrm{~cm}$ & $1 \mathrm{~cm}$ & \\
\hline internal & Horizontal & 0.78 & 0.66 & 0.51 & 0.07 \\
\hline waves & Vertical & 1.98 & 1.69 & 1.33 & 0.49 \\
\hline no internal & Horizontal & 0.78 & 0.65 & 0.50 & 0.04 \\
\hline waves & Vertical & 1.92 & 1.62 & 1.24 & 0.03 \\
\hline
\end{tabular}

Table 2. The accuracy of the seafloor positioning from the rectangular surveying trajectory by using the difference method, with different acoustic ranging accuracy of 1,5 and $7 \mathrm{~cm}$, and with and without the effect of internal waves of short period. Horizontal and Vertical in the table stand for the horizontal and vertical components of the seafloor transponder position, respectively.

\begin{tabular}{|c|c|c|c|c|c|}
\hline \multirow{2}{*}{\multicolumn{2}{|c|}{$\begin{array}{l}\text { Position Accuracy } \\
\text { Ranging Accuracy }\end{array}$}} & \multicolumn{3}{|c|}{ Total MSE $(\mathrm{cm})$} & \multirow{2}{*}{$\begin{array}{l}\text { Systematic } \\
\text { Effect }(\mathrm{cm})\end{array}$} \\
\hline & & $7 \mathrm{~cm}$ & $5 \mathrm{~cm}$ & $1 \mathrm{~cm}$ & \\
\hline \multirow{2}{*}{$\begin{array}{c}\text { internal } \\
\text { waves }\end{array}$} & Horizontal & 2.36 & 2.35 & 2.34 & 2.33 \\
\hline & Vertical & 3.12 & 3.05 & 2.97 & 2.86 \\
\hline \multirow{2}{*}{$\begin{array}{l}\text { no internal } \\
\text { waves }\end{array}$} & Horizontal & 2.36 & 2.35 & 2.33 & 2.32 \\
\hline & Vertical & 3.04 & 2.97 & 2.89 & 2.77 \\
\hline
\end{tabular}

veying strategy can help greatly reduce or cancel out the effect of the remaining systematic errors on seafloor positioning. This is very likely due to the fact that the remaining systematic errors due to the internal waves of short period after difference of measurements behave periodically and can thus be significantly reduced or cancelled out through a symmetrical design of surveying strategy, which looks more or less like the SIO method in cancelling out the effect of systematic errors through a uniform design of transponders and by confining the surveying vessel around the center of the configuration. However, unlike the SIO method, the difference method can also produce the vertical component of a transponder at the accuracy of one or two centimeters. We can also conclude from this table that the accuracy of the horizontal and vertical components is mainly affected by the accuracy of kinematic GPS, since changing the accuracy of acoustic rangings only slightly affect the positioning accuracy.

In the case of the rectangular (B) surveying strategy (compare the right plot of Fig. 4 and Table 2), the effects of the remaining systematic errors are still at the level of 2 to $3 \mathrm{~cm}$ such that they basically dominate the mean squared errors of positioning, although the final accuracy of positioning is about $2.35 \mathrm{~cm}$ in the horizontal components and around $3 \mathrm{~cm}$ in the vertical component. These experiments have shown that (i) both surveying strategies can produce centimeters level of accuracy in seafloor positioning; (ii) the rectangular surveying strategy is less effective in reducing or removing the effects of systematic errors on precise seafloor positioning than the circular surveying strategy. This might be explained as follows: the circular surveying route is horizontally symmetrical with respect to the transponder at every $6 \mathrm{~km}$, while the rectangular route is only so after finishing a complete surveying route. In other words, the circular surveying strategy is some tens times more effective in terms of symmetry than the rectangular one. As a result, the circular surveying strategy is more effective in reducing the effect of residual systematic errors. We may also note that if acoustic signals are much stronger with the advance of technology in the future and are less affected by the noise of engine of the surveying ship, then the ship could move faster. With the decrease of time moving from one point to next, the residual systematic errors should decrease as well; and (iii) a well-designed surveying strategy would be very helpful in improving the accuracy of seafloor positioning.

To investigate the effect of sample spacing on seafloor positioning by the difference method, we will keep the whole scheme of the above experiment unchanged except for that the sampling rate will not be one data every $100 \mathrm{~m}$ but will be one data every 50 to $300 \mathrm{~m}$ along the surveying trajectory. Kinematic GPS and acoustic ranging measurements are assumed to be of 10 and $7 \mathrm{~cm}$, respectively. We have plotted in Fig. 6 the mean squared errors in the horizontal and vertical components of the seafloor transponder and the effects of the systematic errors on seafloor positioning with internal waves of short period. It is obvious from Fig. 6 that the accuracies of the horizontal components are all around $1 \mathrm{~cm}$, while those of the vertical component run from 1.43 to $3.29 \mathrm{~cm}$. The effect of systematic errors is much smaller on the horizontal components than on the vertical component. We can also see that the accuracies of the horizontal and vertical components tend to increase with the increase of sample spacings. This can be well explained by the yellow line of Fig. 6, because the numbers of total points collected have been decreased. Even if we do collect one data every 300 meters, by spending more days on sea and/or by improving the accuracy of acoustic ranging measurements, we would collect more precise data. As a result, we should improve the accuracy of positioning for 


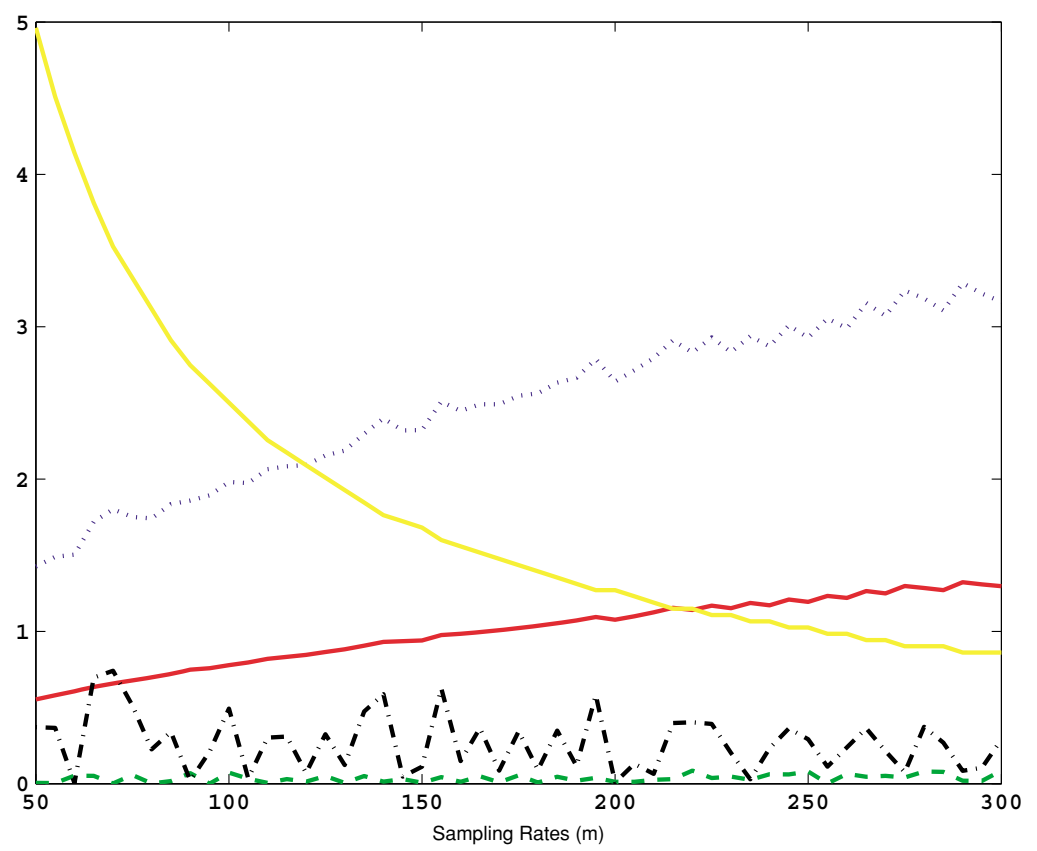

Fig. 6. The effect of sample spacings on seafloor positioning by the difference method, with the effects of internal waves of short period: the horizontal axis - the sample spacings in meters; the red solid line - the accuracy or root mean squared error (MSE) of the horizontal components; the blue dotted line - the accuracy or root MSE of the vertical component; the green dashed line - the systematic effect on the horizontal components due to the systematic errors; the black dash-dotted line-the systematic effect on the vertical component due to the systematic errors; the yellow solid line-the numbers of sample points corresponding to the sample spacings (unit: 1000 points). Except for the yellow line, the units in the vertical axis are all in centimeters for the other four lines.

both horizontal and vertical components to the level of 1 $\mathrm{cm}$.

\subsection{Relative seafloor positioning}

Relative seafloor positioning is to determine the relative position of two transponders on the seafloor by using the acoustic ranging measurements collected by the boat hydrophone. It may be thought of as the simplest example of a seafloor geodetic network without use of the measurements collected by towed hydrophones. If there exist internal waves of short period from minutes to tens of minutes, it is theoretically expected in Section 2.1 and has been shown numerically in Section 5.1.2 (compare Fig. 5) that a significant part of systematic errors of short period nature (internal waves, for example) can still remain in the single difference measurements, and can significantly affect accuracy on seafloor positioning, the extent of which is related to surveying strategies, however. We have also learnt from Section 3.2 that if two consecutive sample points are sufficiently close, spatial and temporal effects of systematic errors can all be removed. Thus we will use the double difference method to determine the relative position between two transponders on the seafloor.

Without loss of generality, we will fix one of two transponders and focus on the determination of the relative vector, which is denoted by $\Delta \mathbf{x}$. Then the observation equations of type (20) can be written as

$$
\nabla \Delta \rho^{(i+1) i}(\Delta \mathbf{x})=\left(\mathbf{a}_{i o_{2}}-\mathbf{a}_{(i+1) o_{2}}\right) \delta \Delta \mathbf{x}+\epsilon_{(i+1) i},
$$

where

$$
\begin{aligned}
\epsilon_{(i+1) i}= & \left(\mathbf{a}_{i o_{1}}-\mathbf{a}_{i o_{2}}\right) \boldsymbol{\epsilon}_{x_{i}}-\left(\mathbf{a}_{(i+1) o_{1}}-\mathbf{a}_{(i+1) o_{2}}\right) \boldsymbol{\epsilon}_{x_{i+1}} \\
& -\epsilon_{i o_{1}}+\epsilon_{i o_{2}}+\epsilon_{(i+1) o_{1}}-\epsilon_{(i+1) o_{2}} .
\end{aligned}
$$

In order to investigate the accuracy of seafloor positioning by the double difference method, we design the circular and rectangular surveying trajectories to measure the relative position between two transponders (Fig. 7). We assume the radius of depth plus half of the vector $\Delta \mathbf{x}$ for the circular trajectory. In the case of rectangular trajectory, the area is equal to the diameter of the circular trajectory multiplied by two depths. In our experiments, we assume that the two transponders are separated by $1 \mathrm{~km}$. As in Section 5.1.2, we use the sampling rate of about 1.667 minutes for the circular surveying trajectory. Since the effect of systematic errors has been almost completely eliminated by the double difference operator, we will focus on the random effect on seafloor positioning. The kinematic GPS will still be assumed to provide the position of the surveying boat at the accuracy of $10 \mathrm{~cm}$, while acoustic ranging measurements between the boat hydrophone and the transponders on the seafloor are of the accuracy of 1,5 and $7 \mathrm{~cm}$, respectively.

We can see from Table 3 that the double difference method is almost completely free of the effect of systematic errors and results in sub-centimeters accuracy in the horizontal components of relative seafloor positioning and about sub-centimeters to one or two centimeters accuracy in the vertical component. Since collecting more data can compensate noisy acoustic ranging measurements, we conclude that the double difference method should be able to determine relative seafloor positioning at the accuracy of sub-centimeters horizontally and vertically. Since the method removes the effect of systematic errors through a double difference operator and is basically irrelevant of the surveying boat speed, if the signals of transponders are strong enough, the field work on sea can be significantly 

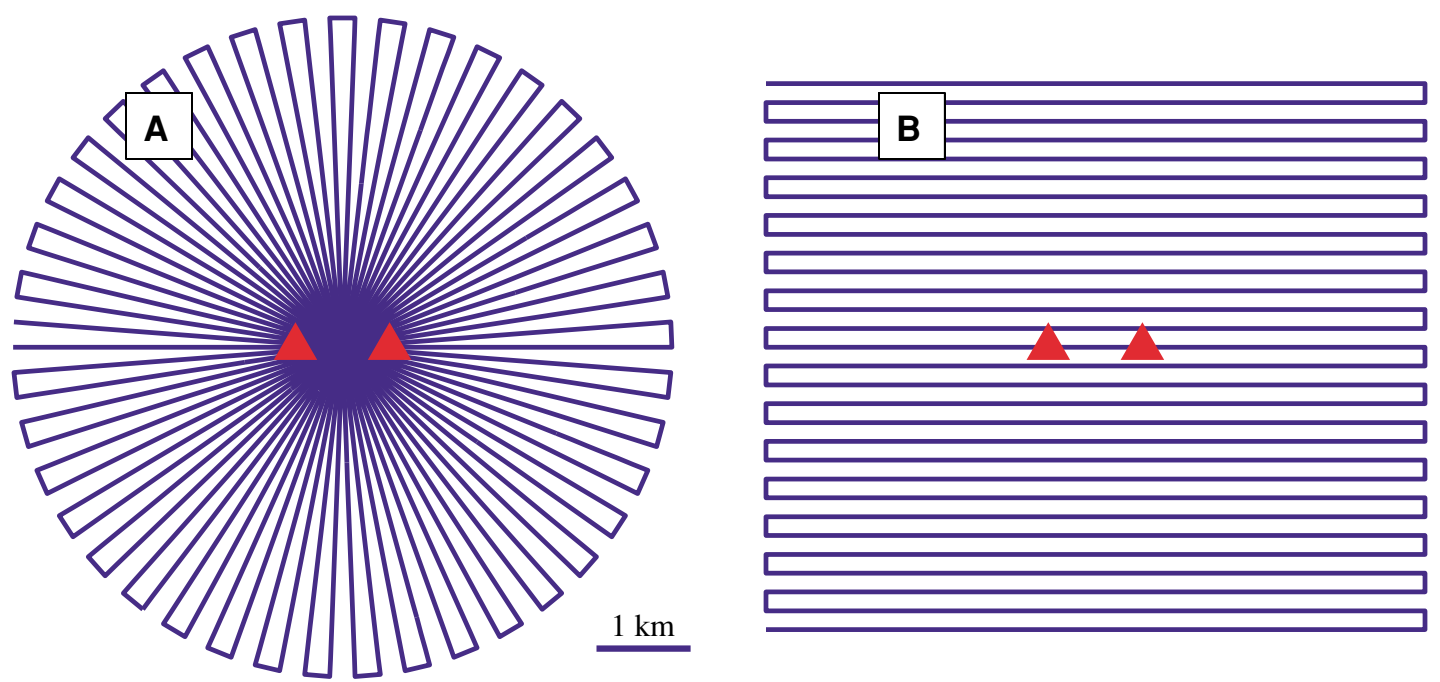

Fig. 7. The two surveying strategies (trajectories) for testing the performance of the double difference method: the solid lines—the surveying trajectories; the red triangles- the transponders on the seafloor.

Table 3. The accuracy of the relative seafloor positioning from the circular (A) and rectangular (B) surveying trajectories by using the double difference method, with different acoustic ranging accuracy of 1,5 and $7 \mathrm{~cm}$. Horizontal and Vertical in the table stand for the horizontal and vertical components of the seafloor transponder position, respectively.

\begin{tabular}{cc|ccc|c}
\hline \multicolumn{2}{c|}{ Position Accuracy } & \multicolumn{3}{c|}{ Total MSE $(\mathrm{cm})$} & \multirow{2}{*}{$\begin{array}{c}\text { Systematic } \\
\text { Effect }(\mathrm{cm})\end{array}$} \\
\hline \multicolumn{2}{c|}{ Ranging Accuracy } & $7 \mathrm{~cm}$ & $5 \mathrm{~cm}$ & $1 \mathrm{~cm}$ & \\
\cline { 1 - 5 } $\begin{array}{c}\text { circular } \\
\text { trajectory }\end{array}$ & Horizontal & 0.79 & 0.57 & 0.15 & \\
\cline { 2 - 5 } $\begin{array}{c}\text { rectangular } \\
\text { trajectory }\end{array}$ & Vertical & 1.79 & 1.29 & 0.36 & \multirow{2}{*}{ Eliminated } \\
\cline { 2 - 5 } & Horizontal & 0.60 & 0.43 & 0.11 & \\
\hline
\end{tabular}

shortened in time to achieve sub-centimeters level of accuracy for relative seafloor positioning. We have also shown in Fig. 8 the variations of accuracy for the circular trajectory at the accuracy of 1,5 and $7 \mathrm{~cm}$ for acoustic ranging measurements. It can be seen that the accuracy of relative seafloor positioning can be as good as sub-centimeters in the horizontal components for all the three cases, and is between 1 and $3 \mathrm{~cm}$ in the vertical component in the cases of 5 and $7 \mathrm{~cm}$, and reaches again sub-centimeters in the case of $1 \mathrm{~cm}$. Even for the former two cases, if more data are collected on sea, sub-centimeters accuracy for the vertical component should also be expected, since this would be equivalent to increasing the accuracy for acoustic ranging measurements.

\section{Concluding Remarks}

Seafloor geodesy has been developed for measuring seafloor crustal deformation by Spiess (1980, 1985a, b), based on the idea of Peter Bender by installing three or four transponders uniformly along a circle on the seafloor and by keeping the surveying vessel as close to the center of the circle as possible. Such a surveying strategy has been shown to be effective in precisely determining the horizontal components of the configuration center, since systematic errors have been cancelled out. However, the effect of systematic errors on the vertical component remains unchanged. The repeatability for the horizontal components obtained by the group of researchers at the Scripps Institution of Oceanography was recently reported to be less than $10 \mathrm{~mm}$ (Gagnon et al., 2005). In regions of local deformation, the SIO method requires the internal geometry of the transponders to be re-determined by using a deeply-towed vehicle.

We have proposed the single difference method for single point seafloor positioning and the double difference method for relative seafloor positioning. The simulations have clearly shown that transponders on the seafloor can be determined at the accuracy of $1 \mathrm{~cm}$ in the horizontal components and $2 \mathrm{~cm}$ in the vertical component by combination of kinematic GPS, acoustic ranging and a properly designed surveying scheme. If systematic errors are of long period nature, they could be removed by the single difference method. However, if there exist systematic errors of short period, for example, internal waves with period of minutes to tens of minutes, a significant part of errors will remain in the single-differenced acoustic ranging measurements. If acoustic signals could be significantly strengthened such that they are less affected by the noise of the ship engine, then the surveying ship could move at a faster speed. As a result, residual systematic errors should be further reduced significantly, depending on the ratio of movement time to the period of internal waves. We have shown that the remaining errors could be further reduced and/or cancelled out by a properly designed surveying scheme. We 


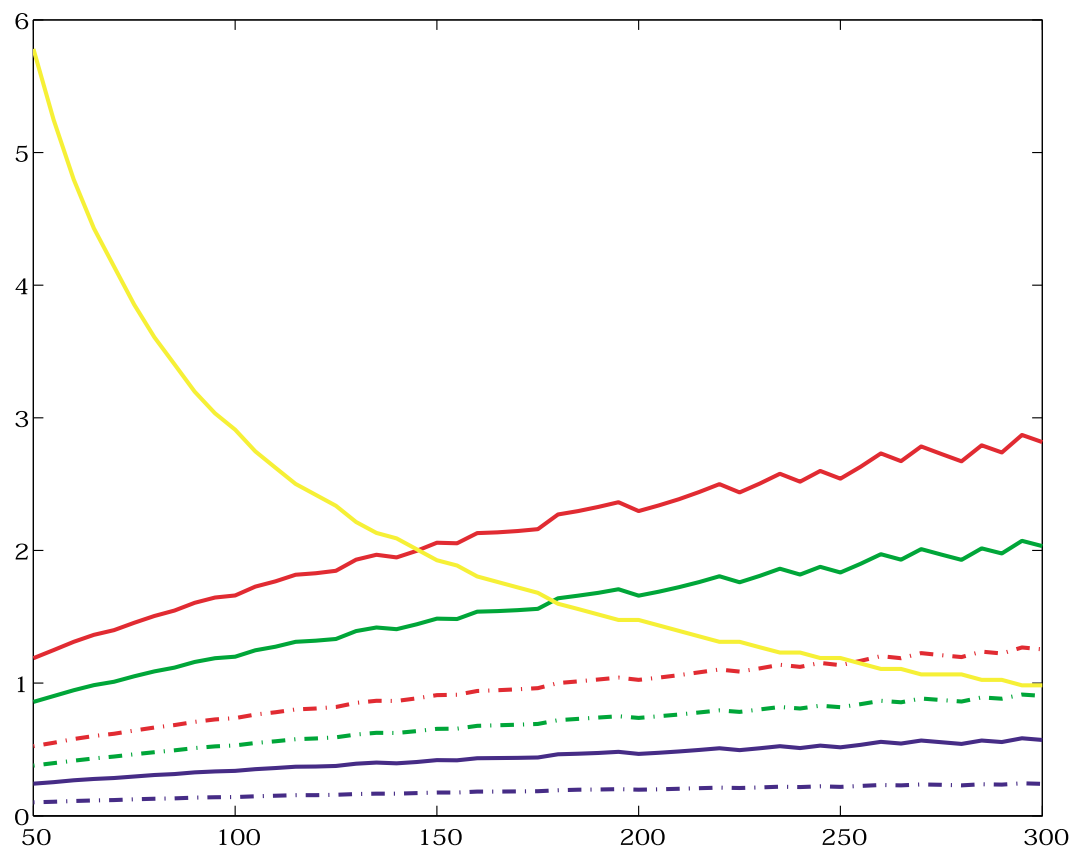

Fig. 8. The effects of sample spacings and acoustic ranging errors on the accuracy of relative seafloor positioning. The horizontal axis shows the sample spacings in meters along the trajectory. The units in the vertical axis, except for the yellow line, are in centimeters. The two red, green and blue lines correspond to the accuracy of $7 \mathrm{~cm}, 5$ and $1 \mathrm{~cm}$ for acoustic ranging measurements, respectively. The dash-dotted and solid lines show the accuracy in the horizontal and vertical components, respectively. The yellow line marks the numbers of sample points for each sample spacing (units: 1000 points).

must note that if the symmetry of surveying scheme is not kept, the remaining part of systematic errors (of short period) may prevent us from determining the transponders at the centimeters accuracy.

We have also conducted the experiments to determine the relative position between two transponders on the seafloor by using the double difference method. Unlike the single difference method, the double difference method is also able to remove the effect of systematic errors of short period, internal waves with period of minutes to tens of minutes, for example, on relative seafloor positioning, if the two consecutive sampled points are sufficiently close. As a result, the double difference method is basically affected only by random errors. The simulations have shown that the three-dimensional, relative position between two transponders separated by $1 \mathrm{~km}$ on the seafloor can be measured at the sub-centimeters level of accuracy, given the accuracy of $10 \mathrm{~cm}$ for kinematic GPS and the accuracy of 1,5 and 7 $\mathrm{cm}$ for acoustic ranging measurements. Although the attained accuracy is derived with the symmetry of surveying scheme, this is not essentially needed by the double difference method. Thus the efficiency of field work on sea can be significantly improved if the signals of transponders are sufficiently strong. If many transponders are installed on the seafloor and, if measurements collected by towed hydrophones are included and processed together with acoustic ranging measurements collected by the on-board hydrophone in double difference mode, we are able to establish a seafloor geodetic network for precise seafloor crustal deformation measurement.

Acknowledgments. The authors thank Dr. M. Fujita for the constructive comments. They are also indebted to an anonymous re- viewer for constructive comments, for polishing the English of the text and for bringing the two recently published articles to their attention. The work of the first author is supported by a Grant-inAid for Scientific Research C(2)16540386.

\section{References}

Anderson, G., S. Constable, H. Staudigel, and F. Wyatt, A seafloor longbaseline tiltmeter, J. Geophys. Res., B102, 20269-20285, 1997.

Ando, M., Source mechanisms and tectonic significance of historical earthquakes along the Nankai Trough, Japan, Tectonophys., 27, 119-140, 1975.

Asada, A. and T. Yabuki, Centimeter-level positioning on the seafloor, Proc. Japan Acad. Ser. B, 77, 7-12, 2001.

Chadwell, D., Shipboard towers for Global Positioning System antennas, Ocean Eng., 30, 1467-1487, 2003.

Chadwell, D., F. Spiess, J. Hildebrand, L. Young, G. Purcell, Jr., and H. Dragert, Deep-sea geodesy: Monitoring the ocean floor, GPS World, 9 , 44-55, 1998.

Cohen, C. E., Attitude determination, in Global Positioning System: Theory and Applications, Vol. II, edited by B. W. Parkinson, J. J. Spilker, Jr., P. Axelrad and P. Enge, pp. 519-538, Amer. Inst. Aeronautics \& Astronautics, Washington, 1996.

Colosi, J. A., S. M. Flatté, and C. Bracher, Internal-wave effects on 1000$\mathrm{km}$ oceanic acoustic pulse propagation: Simulation and comparison with experiment, J. Acoust. Soc. Am., 96, 452-468, 1994.

Desaubies, Y., Ocean acoustic tomography, in Oceanographic and Geophysical Tomography, edited by Y. Desaubies, A. Tarantola, and J. ZinnJustin, pp. 159-202, Elsevier, Amsterdam, 1990.

Flatté, S. M. and M. D. Vera, Internal-wave time evolution effect on ocean acoustic rays, J. Acoust. Soc. Am., 112, 1359-1365, 2002.

Flatté, S. M., R. Dashen, W. H. Munk, K. M. Watson, and F. Zachariasen, Sound Transmission trough a Fluctuating Ocean, Cambridge University Press, Cambridge, 1979.

Frank, F. C., Deduction of earth strains from survey data, Bull. Seismol. Soc. Am., 56, 35-42, 1966.

Fujimoto, H., T. Kanazawa, and H. Murakami, Experiment on precise seafloor acoustic ranging-A promising result of observation, J. Seismol. Soc. Japan, 48, 289-292, 1995 (in Japanese).

Fujimoto, H., K. Koizumi, Y. Osada, and T. Kanazawa, Development of instruments for seafloor geodesy, Earth Planets Space, 50, 905-911, 
1998.

Gagnon, K., C. D. Chadwell, and E. Norabuena, Measuring the onset of locking in the Peru-Chile trench with GPS and acoustic measurements, Nature, 434, 205-208, 2005.

Gordon, R. G. and S. Stein, Global tectonics and space geodesy, Science, 256, 333-342, 1992.

Lambeck, K., Geophysical Geodesy: The Slow Deformations of the Earth, Clarendon Press, Oxford, 1988.

Lurton, X., An Introduction to Underwater Acoustics, Springer, London, 2002

Obana, K., H. Katao, and M. Ando, Seafloor positioning system with GPSacoustic link for crustal dynamics observation-a preliminary result from experiments in the sea, Earth Planets Space, 52, 415-423, 2000.

Prescott, W. H., The determination of displacement fields from geodetic data along a strike slip fault, J. Geophys. Res., B86, 6067-6072, 1981.

Purcell, G. H., L. E. Young, S. K. Wolf, T. K. Meehan, C. B. Duncan, S. S. Fisher, F. N. Spiess, G. Austin, D. E. Boegeman, C. D. Lowenstein, C. Rocken, and T. M. Kelecy, Accurate GPS measurement of the location and orientation of a floating platform, Mar. Geod., 14, 255-264, 1990.

Shimamura, H. and T. Kanazawa, Ocean bottom tiltmeter with acoustic data-retrieval system implanted by a submersible, Mar. Geophys. Res., 9, 237-254, 1988 .

Spiess, F. N., Acoustic techniques for marine geodesy, Mar. Geod., 4, 1327, 1980 .
Spiess, F. N., Suboceanic geodetic measurements, IEEE Trans. Geosc. Remote Sens., GE-23, 502-510, 1985a.

Spiess, F. N., Analysis of a possible sea floor strain measurement system, Mar. Geod., 9, 385-398, 1985b.

Spiess, F. N., D. Chadwell, J. A. Hildebrand, L. E. Young, G. H. Purcell, Jr., and H. Dragert, Precise GPS/acoustic positioning of seafloor reference points for tectonic studies, Phys. Earth Planet. Int., 108, 101-112, 1998.

Sweeney, A. D., C. D. Chadwell, J. A. Hildebrand, and F. N. Spiess, Centimeter-level positioning of seafloor acoustic transponders from a deeply towed interrogator, Mar. Geod., 28, 39-70, 2005.

Webb, S. C., Broadband seismology and noise under the ocean, Rev. Geophys., 36, 105-142, 1998.

Webb, S. C., T. K. Deaton, and J. C. Lemire, A broadband ocean-bottom seismometer system based on 1-Hz natural period geophone, Bull. Seismol. Soc. Am., 91, 304-312, 2001

Yamada, T., M. Ando, K. Tadokoro, K. Sato, T. Okuda, and K. Oike, Error evaluation in acoustic positioning of a single transponder for seafloor crustal deformation measurements, Earth Planets Space, 54, 871-881, 2002.

P. Xu (e-mail: pxu@rcep.dpri.kyoto-u.ac.jp), M. Ando, and K. Tadokoro 